\title{
High Temperature Measurement System Design for Thermoelectric Materials In Power Generation Application
}

\author{
Sim Loo ${ }^{\dagger}$, Jarrod Short ${ }^{\dagger}$, Kuei Fang Hsu ${ }^{\ddagger}$, Mercouri Kanatzidis ${ }^{\ddagger}$, Tim Hogan ${ }^{\dagger}$ \\ ${ }^{\dagger}$ Electrical and Computer Engineering Department, Michigan State University \\ 2120 Engineering Building, East Lansing, MI 48824-1226 \\ Chemistry Department, Michigan State University \\ East Lansing, MI 48824-1322
}

\begin{abstract}
Recent interest in thermoelectric materials for power generation applications has initiated the development of a measurement system in our laboratory for characterization of materials in the $80 \mathrm{~K}$ to $800 \mathrm{~K}$ temperature range. This system has been specifically designed for measuring thermoelectric power and electrical conductivity as needed for determining the power factor of the measured samples. This is a single sample measurement system based on a continuous flow cryostat. Significant effort has gone into the computer controlled data acquisition and PID controlled temperature stabilization. Investigation of the influence of temperature stability on the measured data will be presented along with important aspects of the system design, development, and testing. Data collected on reference materials and new thermoelectric materials of interest will be presented.
\end{abstract}

\section{INTRODUCTION}

A high temperature (HT) thermoelectric measurement system was fabricated and the detailed system description can be found in [1]. The system is designed for measuring thermoelectric power, and electrical conductivity of any electronic material. This paper is intended to present how this system is used as a tool to investigate new cubic materials which are fabricated at Michigan State University (MSU). This system was also used for characterizing a TE module made up of such new materials. The figure of merit of the module was determined through the thermal conductance measurement technique.

\section{THEORY OF THERMAL CONDUCTANCE MEASUREMENT TECHNIQUE}

The thermal conductance measurement technique is a method for determining the $Z T$ from thermal conductance measurements on a thermoelement that is connected in parallel with a metallic wire [2]. The thermal conductance $K$ of a sample, in the absence of an electric field ( $E=$ $0)$ is $K_{E=0}$ and under zero current flow $(I=0)$, is $K_{I=0}$, it can be found that

$$
K_{E=0}=K_{I=0}(1+z T)
$$

where $z$ is the figure of merit of a single element. The heat equation is expressed as

$$
Q=K \Delta T+S_{p n} T_{h} I-\frac{1}{2} I^{2} R
$$

where $Q$ is the heat flow, $\Delta T$ is the temperature gradient, $K, S$ and $R$ are the thermal conductance, Seebeck coefficient, and resistance of the thermoelement, respectively. 


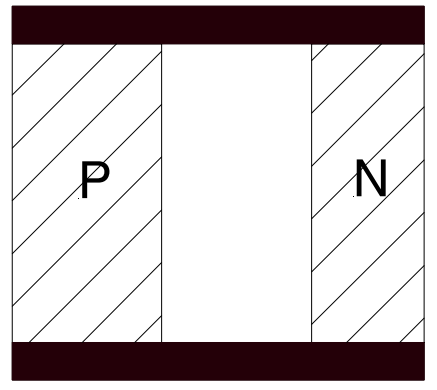

Figure 1 Short circuited TE module with $\mathrm{p}$ and n-legs.

This concept can also be applied to a module. Figure 1 shows a module short circuited, where the voltage is zero, i.e. the electric field is zero at the output of the module. The short circuit heat flow is

$$
Q_{\text {short }}=K_{p n} \Delta T+S_{p n} T_{h} I-\frac{1}{2} I^{2} R_{\text {Module }}
$$

where $R_{\text {Module }}$ is the resistance of the $p$ and $n$-legs plus the contact resistance.

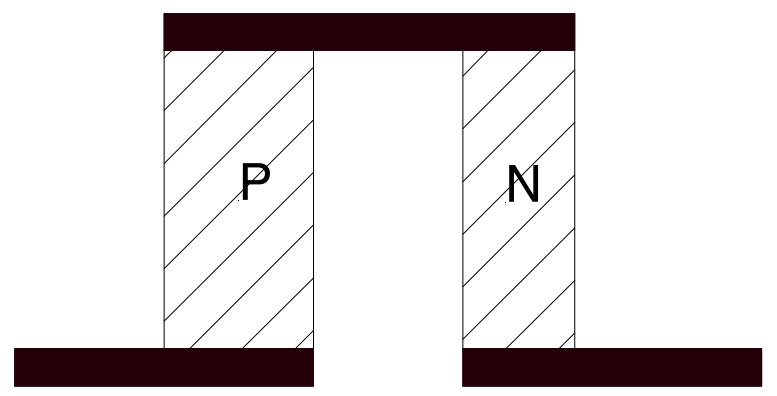

Figure 2 Open circuited TE module with $p$ and $n$-legs.

Figure 2 shows an open circuit module where there is no current flow and the heat flow from the top to the bottom of the module is

$$
Q_{\text {open }}=K_{p n} \Delta T
$$

When a temperature gradient is created across the module, there will be a flow of current. It will lead to heat transfer through the Peltier effect. The current is equal to $\left(S_{p n}\right) \Delta T /\left(R_{\text {module }}\right)$ and the Peltier heat flow is $\left(S_{p n}\right)^{2} T \Delta T /\left(R_{\text {module }}\right)$. Substitute $I$ in equation 3 with $\left(S_{p n}\right) \Delta T /\left(R_{\text {module }}\right)$. The short circuit equation is then

$$
\frac{Q_{\text {short }}}{\Delta T}=K_{p n}+\frac{S_{p n}^{2}}{R_{\text {module }}}\left(T_{h}-\frac{1}{2} \Delta T\right)=K_{p n}+\frac{S_{p n}^{2}}{R_{\text {mod } u l e}} T_{M}
$$

where $T_{M}$ is the mean temperature. Comparing equations 4 and 5 gives

$$
K_{p n, E=0}=K_{p n, I=0}(1+Z T)
$$

By measuring the thermal conductance of the module with its input leads separated and then short circuited, the $Z T$ of the module can be determined. This method can be applied to whole modules as well as to individual thermocouples. 


\section{EXPERIMENTAL SETUP}

The high temperature system consists of a cryostat chamber, a sample stage with a heat shroud and a collection of measurement instruments. The sample is mounted with two singleended Chromel-Constantan thermocouples. They are used for voltage and temperature measurement. A small temperature gradient is established when a current is passed through the heater which is mounted on top of the sample. The standard 4-point probe method is used for the electrical conductivity measurements.

\section{Temperature stabilization}

The system's temperature is monitored by a temperature controller using PID control. PID control is a simple form of feedback and its control algorithm is by far the most widely used automatic control technique in various industries. In order for the control loop to work properly, the PID loop must be properly tuned.

The PID control algorithm comprises three elements: Proportional (P) - also known as Gain, Integral (I) - also known as Automatic Reset or simply Reset, and Derivative (D) - also known as Rate. The algorithm is normally available in several combinations of these elements. There are various PID tuning methods and every system has its most suitable tuning method.

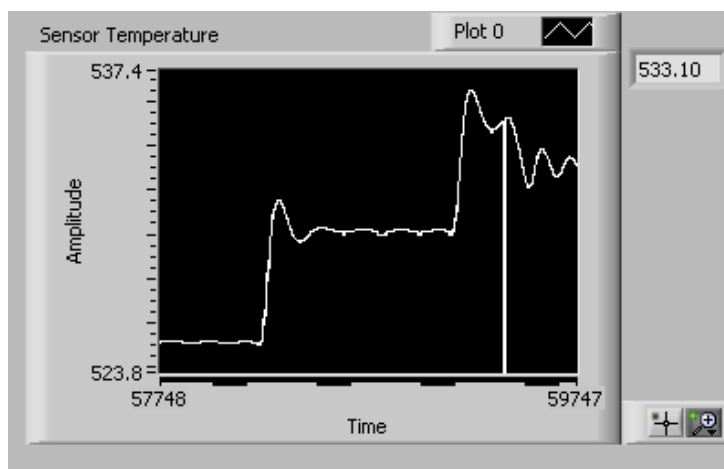

a)

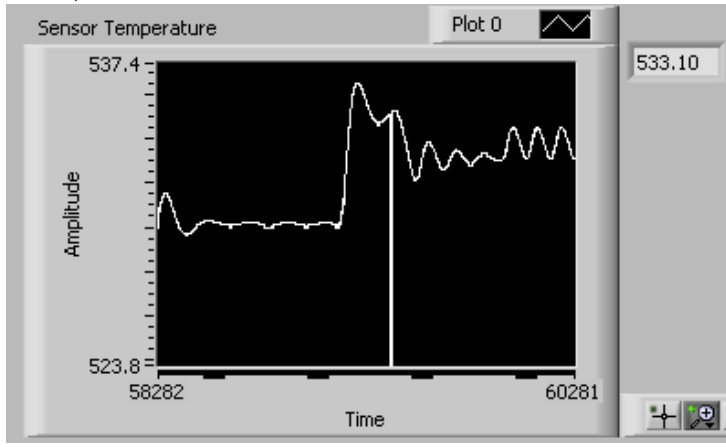

c)

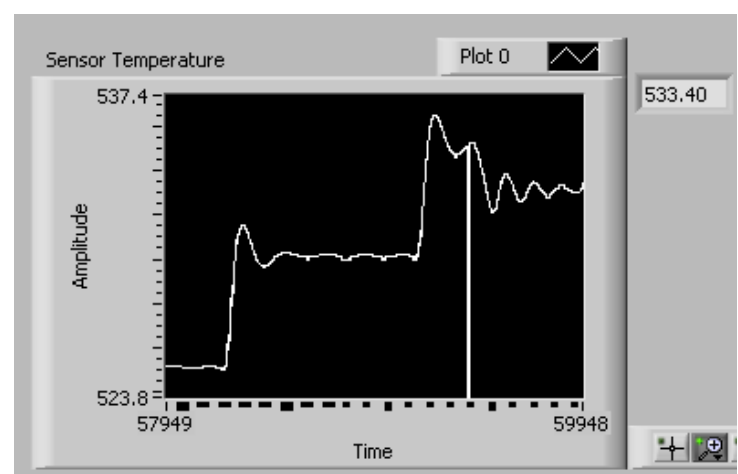

b)

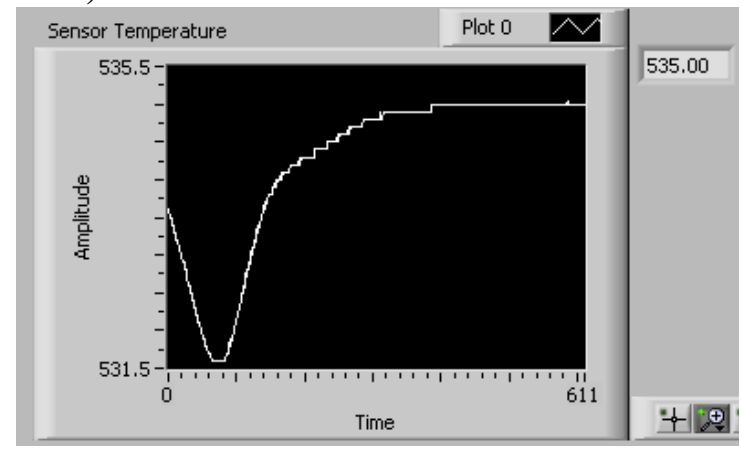

d)

Figure 3 a) When the temperature is not stable, the PID values need to be re-tuned. Set the P value to about 3 times the old $\mathrm{P}$ value and set I and D to zero. b) The amplitude of oscillation decreases. c) Set the $\mathrm{P}$ value to a higher value to obtain an oscillation of constant magnitude. $\mathrm{d}$ ) Retune the PID based on the period of the oscillation and the temperature stabilizes in minutes. 
We have adapted the tuning procedures from the LakeShore Model 330 Autotuning Temperature Controller User's Manual [3] for our high temperature system. A typical tuning procedure is shown in Figure 3.

When tuning the PID of a system, first set the P value to 50 or 100 or a multiple of that and set the I and D value to zero. The purpose of this is to create an oscillation with constant amplitude and utilize the oscillation period to determine the I and D values. Sometimes, retuning PID is necessary during the process of measurement if the system temperature starts to oscillate. Figure 1 a) shows the temperature of the system oscillating. The system was retuned by setting the $\mathrm{P}$ value to three times the old $\mathrm{P}$ value as well as I and $\mathrm{D}$ to zero. The white line on the figure indicates the change of PID. Figure $1 \mathrm{~b}$ ) shows that the amplitude of the oscillation decreases. The $\mathrm{P}$ value was adjusted to obtain constant oscillation amplitude as shown in Figure $1 \mathrm{c})$. The period of the oscillation was used to determine the I and D values. Figure $1 \mathrm{~d}$ ) shows the system stabilized in minutes with the new PID values.

\section{EXPERIMENTAL RESULTS $\underline{\text { Materials }}$}

The system is used to investigate the new cubic materials which had been developed in MSU. These materials consist of lead, antimony, silver and tellurium so they are called the LAST series. Many LAST samples are characterized in this HT system and show very promising results at high temperatures [4]. Generally, the electrical conductivity of these materials decreases with increasing temperature and the magnitude of the thermoelectric power increases linearly with increasing temperature. Most of them exhibit very high power factor (higher than 20) peaking in the temperature range of $300-600 \mathrm{~K}$.

After many cubic materials are characterized, a pair of $\mathrm{p}$ - and n-type samples was selected with comparable properties as function of temperature, to be fabricated into module. Figures 4 and 5 show the electrical conductivity, thermoelectric power and power factor of an ntype sample. The electrical conductivity decreases with increasing temperature and the magnitude of the thermoelectric power increases linearly with temperature. At $500 \mathrm{~K}$, the electrical conductivity is $180 \mathrm{~S} / \mathrm{cm}$ and the thermopower is $-240 \mu \mathrm{V} / \mathrm{K}$. The power factor is approximately $10.36 \mu \mathrm{W} / \mathrm{cm} \cdot \mathrm{K}^{2}$ at $500 \mathrm{~K}$. Figure 6 and 7 show the properties of the p-type sample used. The magnitude of the transport properties of this sample is similar to the n-type sample with the electrical conductivity of $190 \mathrm{~S} / \mathrm{cm}$ and the thermopower is $250 \mu \mathrm{V} / \mathrm{K}$ at $500 \mathrm{~K}$. The power factor is about $11.8 \mu \mathrm{W} / \mathrm{cm} \cdot \mathrm{K}^{2}$ at $500 \mathrm{~K}$. As mentioned above, higher power factors have been achieved in these materials, however, we chose $n$ and $p$-type samples with comparable properties for this paper. 


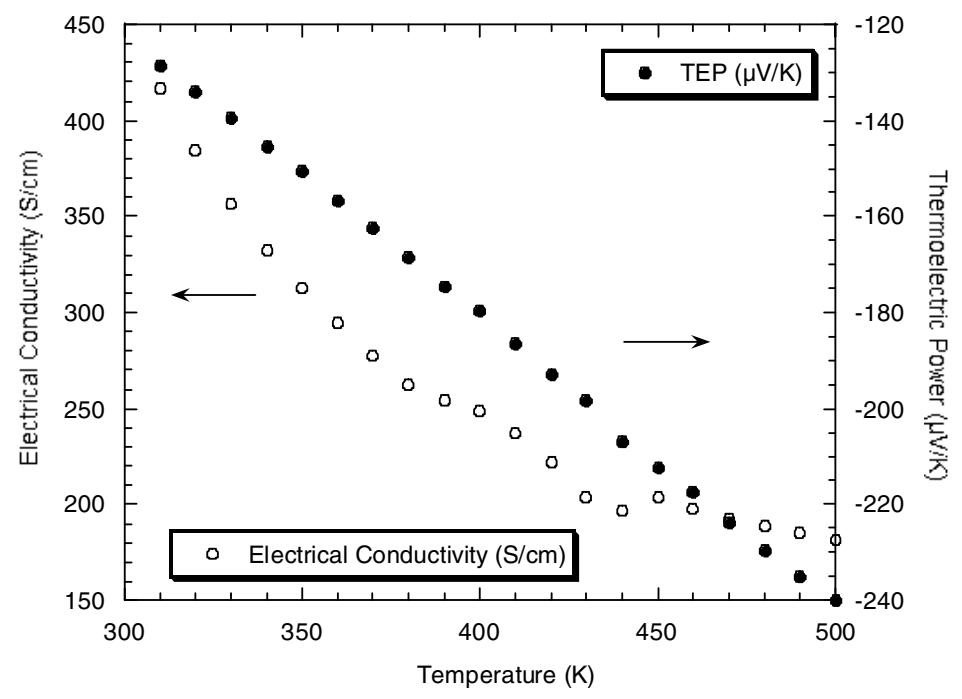

Figure 4 The electrical conductivity and thermopower of n-type sample KF2228R4A2

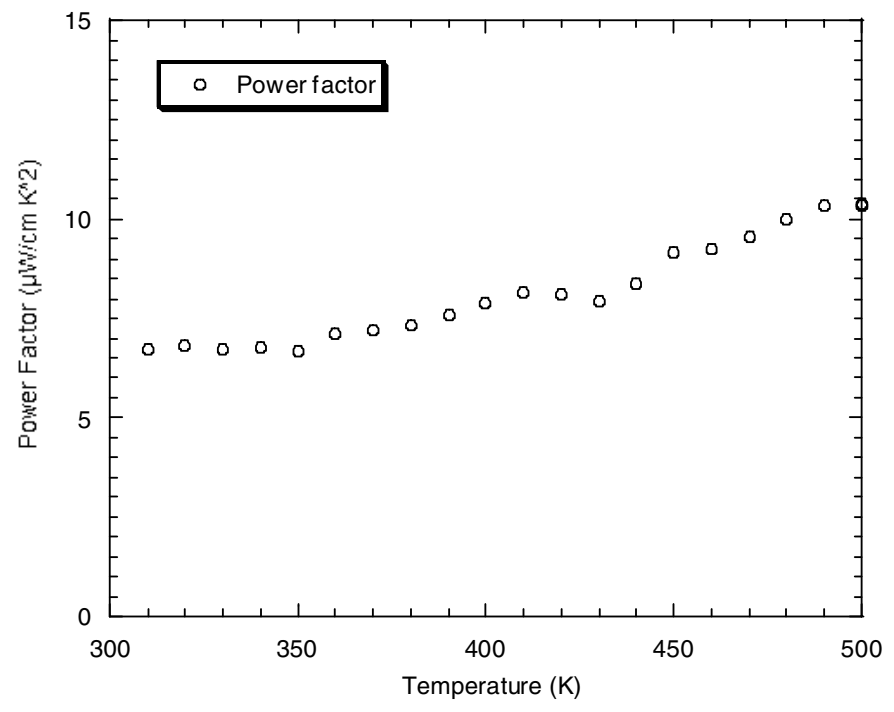

Figure 5 The power factor of n-type sample KF2228R4A2 


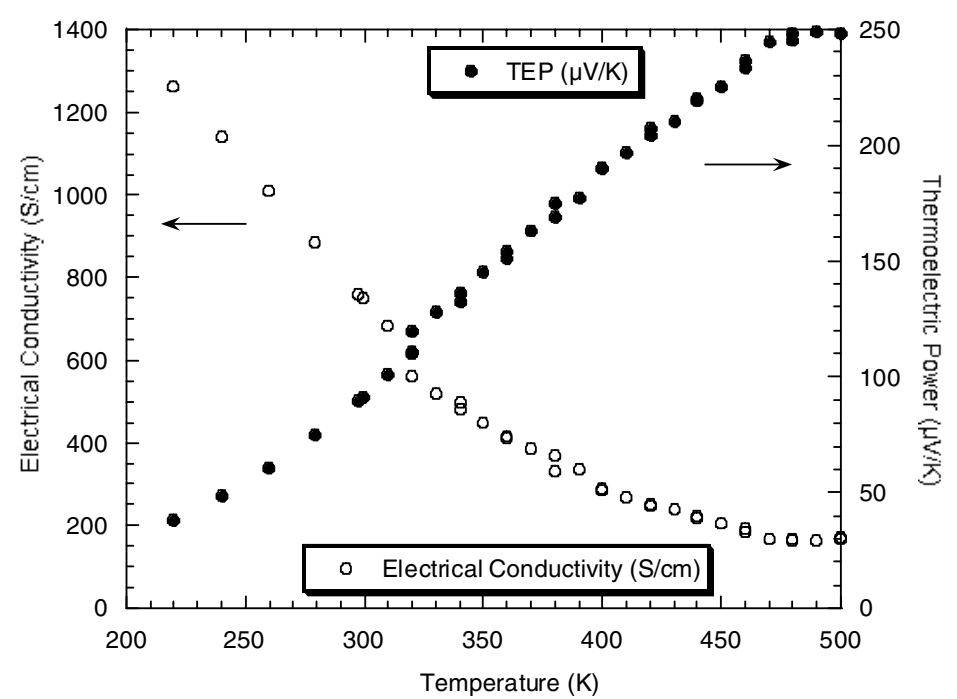

Figure 6 The electrical conductivity and thermopower of p-type sample KF2250R2

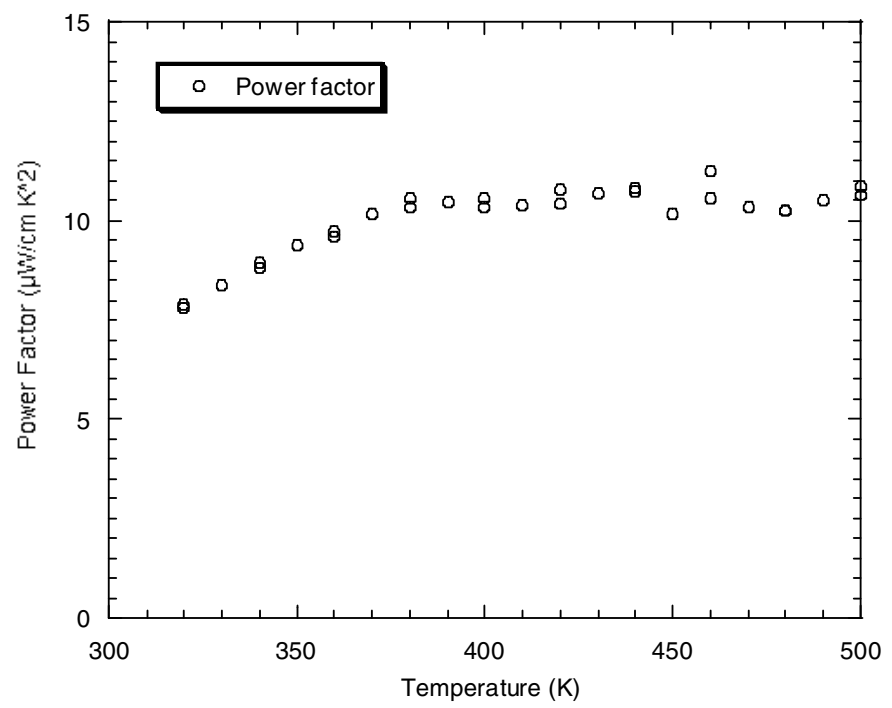

Figure 7 The power factor of p-type sample KF2250R2

\section{$\underline{\text { Module }}$}

The performance of a TE power generator depends on the hot side temperature, $T_{h}$, the temperature difference between the hot and cold ends, $\Delta T$, and the Figure of Merit of the materials, $Z T$. The efficiency of a single-couple TE generator without contact resistance is given by

$$
\eta_{t}=\frac{W_{e}}{Q_{h}} \quad \text { or } \quad \eta_{t}=\frac{\mu \eta_{c}}{\left[(\mu+1)^{2} / Z T_{H}+(\mu+1)-\eta_{c} / 2\right]}
$$

A TE module made up of an $n$-type cubic material, KF2228R4A2, and a $p$-type cubic material, KF2250R2, was fabricated. Both samples have similar thermoelectric efficiency. The 
$n$-type cubic materials have been heavily investigated as discussed in [4]. The optimized material was not used to fabricate the module because an optimized $p$-type material has not yet been discovered. Therefore, the $n$-type sample that was used in the module was selected to match the $Z T$ of the $p$-type samples that we have.

Figure 8 shows the TE module made of an $n$-type cubic material, KF2228R4A2, and a $p$ type cubic material, KF2250R2. The $p$ and $n$-type samples are nickel plated at both ends and sandwiched between two alumina plates with nickel traces. The samples are silver pasted to the top and bottom alumina plates. The total resistance including the contact resistance of the module was tested to be about $0.3 \Omega$ by a handheld multimeter.

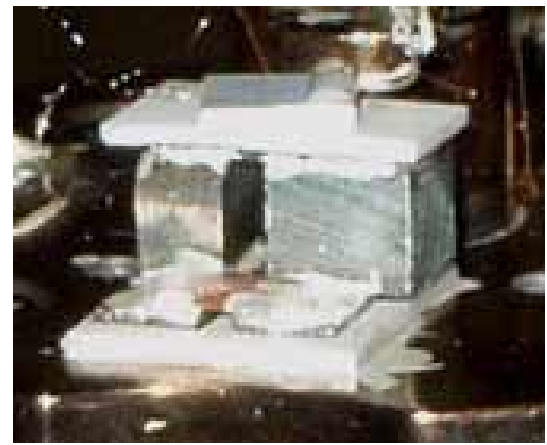

Figure 8 TE module under short circuit test.

The thermal conductance measurement technique, which is described in the Theory section, was used to characterize the module in the same HT system. The module was mounted on the sample stage with silver paste and the system was maintained in a vacuum environment of $10^{-5}$ Torr with the temperature of the sample stage held constant at $300 \mathrm{~K}$. Two single-ended Chromel-Constantan thermocouples were used to monitor the temperature gradient across the module. A platinum RTD was used as a heater and mounted on top of the module to establish the desired temperature gradient. The pulse technique was used to obtain the temperature gradient. Two voltage leads were added to the RTD heater to measure the power applied to the heater. The temperature of the sample stage was maintained at $300 \mathrm{~K}$ with some flow of liquid nitrogen through the cryostat. The PID temperature control was easily tuned to maintain a constant temperature.

First, the thermal conductance of the open-circuit module was measured with various source currents (or temperature gradients). Then the thermal conductance of the short-circuit module was measured. For short-circuit measurements, each of the bottom nickel traces was silver pasted to a copper plate ( $1 \mathrm{~mm}$ diameter). Thermal conductance is $K=\frac{Q}{\Delta T}$, where $\mathrm{Q}$ is the power supplied to the heater. Table 1 shows the experimental results of the open circuit test with various heater currents. The temperature gradients (in volts) obtained by these various heater currents are shown in Figure 9. For a short circuit test with a heater current of $50 \mathrm{~mA}$, the temperature gradient is $356.63 \mathrm{~K}$ and the thermal conductance is $8.726 \mathrm{~mW} / \mathrm{K}$. Figure 10 shows the temperature gradient of open and short circuit tests at $50 \mathrm{~mA}$. Using equation (6), we obtained a $Z T$ of 0.06 for this module at an average temperature of about $330 \mathrm{~K}$. 


\begin{tabular}{|l|l|l|l|}
\hline $\begin{array}{l}\text { Heater current } \\
(\mathrm{mA})\end{array}$ & $\begin{array}{l}\text { Temperature } \\
\text { Gradient }(\mathrm{K})\end{array}$ & $\begin{array}{l}\text { Power Supplied } \\
\text { to Heater }(\mathrm{W})\end{array}$ & $\begin{array}{l}\text { Thermal Conductance } \\
(\mathrm{mW} / \mathrm{K})\end{array}$ \\
\hline 20 & 20.48 & 0.237 & 11.6 \\
\hline 30 & 65.582 & 0.616 & 9.39 \\
\hline 40 & 162.47 & 1.3855 & 8.527 \\
\hline 50 & 390.56 & 3.2165 & 8.2356 \\
\hline
\end{tabular}

Table 1 The experimental result of the open circuit test.

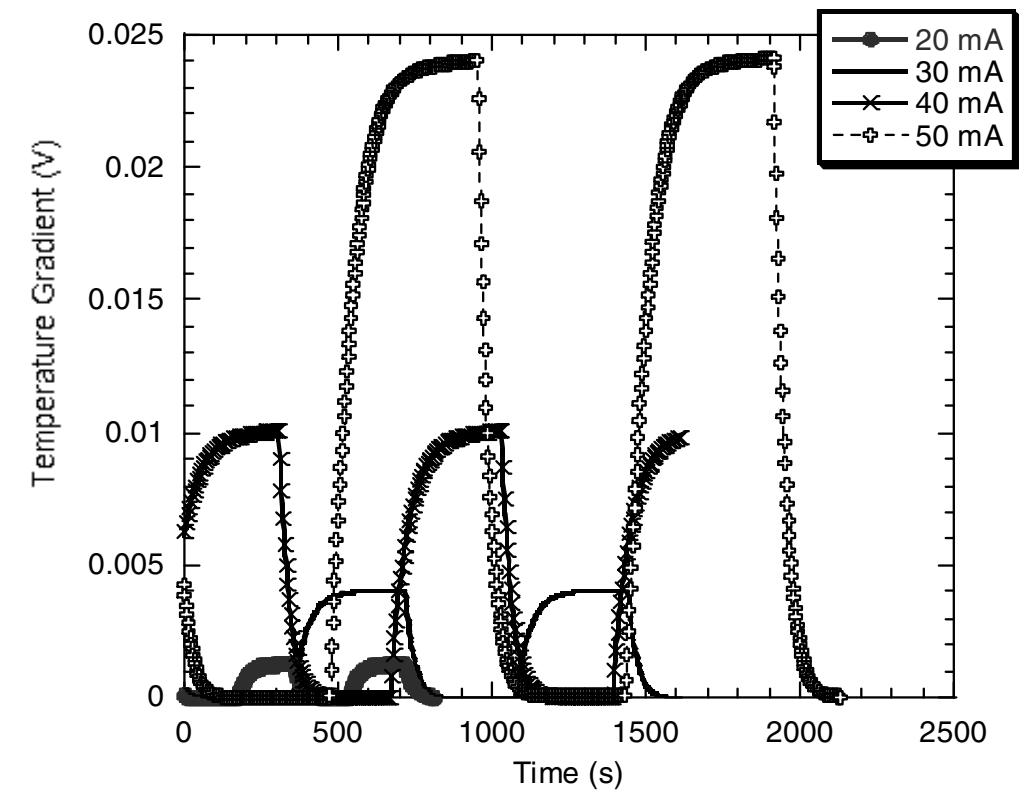

Figure 9 The temperature gradient in volts vs. time with various heater currents applied to the open circuit test.

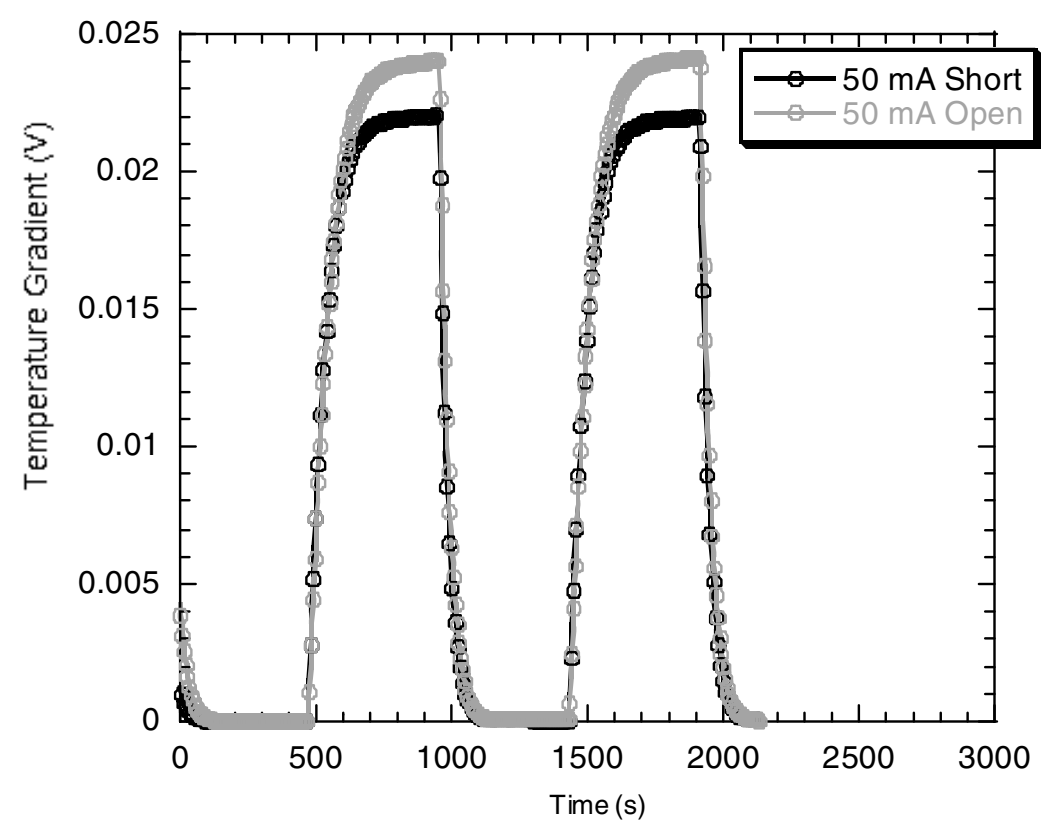

Figure 10 Comparison between open and short circuit at heater current $50 \mathrm{~mA}$. 


\section{CONCLUSION}

The HT measurement system has helped to investigate new cubic materials by determining their power factor. It is also used to investigate the performance of a TE module with the thermal conductance technique. The ZT of the module is low, about 0.06 , because these samples do not show the best properties. Contact resistance between the samples and alumina plates will be investigated as an improvement to the fabrication of the module.

\section{ACKNOWLEDGMENT}

We would like to thank Office of Naval Research (ONR) for the financial support of this project through grant N00014-03-1-0789.

\section{REFERENCES}

[1] S. Loo and T. Hogan, publication in process.

[2] T. A. Al-Obaidi and H. J. Goldsmid, "Determination of the Thermoelectric Figure of Merit from Thermal Conductivity Measurements," Energy Conversion, Vol. 9, pp. 131$132,1969$.

[3] LakeShore User's Manual Model 330 Autotuning Temperature Controller, 11 August 1998, Chapter 3.3.5 Manual control settings (PID), pg. 3-10 to 3-11.

[4] S. Loo, T. P. Hogan, "High Temperature Power Factor Measurement System for Thermoelectric Materials," 204 ${ }^{\text {th }}$ Electrochemical Society conference, October 12-16 2003, Orlando, FL. 